Michał Siedlaczek ${ }^{1}$, Krzysztof Pstragowski ${ }^{1}$, Jakub Ratajczak ${ }^{1}$, Małgorzata Jasiewicz ${ }^{1}$, Klaudyna Grzelakowska1 ${ }^{10}$, Jacek Kryśs ${ }^{2}$, Jacek Kubica ${ }^{1}$

${ }^{1}$ Department of Cardiology and Internal Medicine, Nicolaus Copernicus University, Collegium Medicum in Bydgoszcz, Poland

${ }^{2}$ Antoni Jurasz University Hospital No.1, Nicolaus Copernicus University in Torun, Ludwik Rydygier Collegium Medicum in Bydgoszcz, Poland

\title{
Cost-effectiveness of levosimendan in patients with exacerbation of chronic heart failure - a single-center perspective
}

\section{Corresponding author:}

Małgorzata Jasiewicz, Department of Cardiology and Internal Medicine University Hospital No. 1 ,

ul. M. Sklodowskiej-Curie 9, 85-094 Bydgoszcz, Poland, e-mail: jasiewiczm@gmail.com
Medical Research Journal 2021; Volume 6 , Number 2, 114-118 DOI: $10.5603 /$ MRJ.2021.0027 Copyright (C) 2021 Via Medica ISSN 2451-2591 e-ISSN 2451-4101

\begin{abstract}
Introduction: Heart failure (HF) places a significant economic burden on the health care system all over the world mainly due to frequent and repetitive hospitalizations. Thus, there is a need for both cost-effective and efficient therapeutic options. The aim of the study was to describe the economic aspect of levosimendan treatment in hospitalized HF patients in one major Polish cardiology unit.

Material and methods: Retrospective observational study included 1086 patients with exacerbation of chronic HF, admitted to the Cardiology Department, University Hospital No 1 in Bydgoszcz, Poland in 2018-2020. We analyzed the cost of therapy, cost and the length of stay of 1057 hospitalizations of patients receiving standard therapy (ST) only and 29 hospitalizations of patients receiving levosimendan (Levo) on top. Levosimendan was used in patients not responding to standard therapy, mostly as a drug of the last chance. Results: The mean length of hospital stay for the ST patients was 9.4 days compared to 29.1 days for the Levo group. The median total cost of hospitalization of patients receiving ST was significantly lower compared to Levo group [PLN 6,612.5 (IQR 3,624.9 -13,301.3) vs PLN 23,854.9 (IQR 10,900.4-40,391.5), $\mathrm{p}<0.001]$. On the other hand, the median daily cost of hospitalization did not differ between the ST and Levo group [PLN $772(616.4-1,629)$ vs PLN 1,010.5 (IQR 787.4-1,172), $p=0.1]$. The total cost of treatment was significantly lower only in the ST subgroup hospitalized for less than 2 weeks compared to the Levo group ( $p=0.008$ ). An early decision of levosimendan introduction (up to 8 days) resulted in a shorter hospitalization time compared to later drug administration (21 days vs 42 days; $p=0.019$ ).

Conclusions: Early administered levosimendan in HF exacerbation seems to be cost-effective in Polish clinical and economic settings. Despite the high cost of drug acquisition, it may provide better outcomes at lower overall costs of HF patient management. A randomized trial will be necessary to address this issue in Poland. Key words: decompensated heart failure, cost, levosimendan
\end{abstract}

Med Res J 2021; 6 (2): 114-118

\section{Introduction}

Despite various treatment options available, heart failure (HF) remains a significant public health problem, with an incidence of up to 37.7 million patients worldwide [1]. It significantly affects life expectancy and deteriorates the quality of life. Moreover, it contributes to the high costs for the health care system. Exacerbation of chronic HF is one of the most common causes of patient rehospitalization that significantly rises health expenditure. Hospitalizations for HF account for $1-2 \%$ of all hospital admissions. It is estimated that the total cost of treating patients with HF in the USA will increase from USD 20.9 billion in 2012 to USD 53.1 billion by 2030 [2]. According to data analysis [3], in 2012 the total cost of treating patients with heart failure added up to 108 billion dollars, $60 \%$ of which was allocated directly to treatment costs. 
Moreover, the treatment of HF remains a substantial challenge for health providers, aimed at improving symptoms and reducing mortality of these patients. The management of HF often requires the administration of multiple agents that act on different processes. Levosimendan - an inodilator introduced recently to clinical practice seems to be the one that compromises multiple desired effects on HF exacerbation. In patients with HF, where the positive inotropic effect and the vasodilating capacity are important, the drug increases the force of heart contraction with reduced preload and afterload without increasing myocardial oxygen demand [4], irrespectively to previous beta-blockers intake. The randomized trials have shown that the drug is effective in reducing the mortality of HF patients [5-7]. Unfortunately, the acquisition cost of the levosimendan is relatively high when compared to other standard drug regimens, eg. dobutamine. Several studies have described the cost-effectiveness of levosimendan therapy in HF patients compared to standard options [8-10]. However, the costs of hospitalization due to HF vary from country to country.

Taken into account a significant health and economic burden of HF on society, there is a need for cost-effective therapeutic options that improve symptoms, reduce rehospitalizations and in-hospital mortality of HF patients. The aim of our study was to describe the economic aspect of levosimendan therapy of HF patients from the perspective of a major Polish cardiology unit. Our analysis was prepared as a pre-trial to build the premises addressed for a randomized clinical trial evaluating the cost-effectiveness of early administration of levosimendan in HF exacerbation.

\section{Material and methods}

In this single-center retrospective observational study we included 1086 patients with exacerbation of chronic HF, admitted to the Cardiology Department, University Hospital No 1 in Bydgoszcz, Poland. Patients were hospitalized from 1 January 2018 to 31 December 2020. Exacerbation of HF was diagnosed and managed in accordance with dedicated guidelines of the European Society of Cardiology. The exact costs of each hospitalization were obtained from a dedicated hospital information system. We analyzed the cost of therapy, cost of hospitalization, the length of stay in 1057 hospitalizations of patients receiving standard therapy (ST) for HF exacerbation and 29 hospitalizations of patients receiving levosimendan (Levo). The analysis excluded patients who underwent invasive procedures such as percutaneous coronary intervention (PCI) or implantation of a cardioverter-defibrillator (ICD) during the hospitalization.
The routine practice of introducing levosimendan in HF exacerbation approved in our center was the situation of the inefficacy of ST, including inotropic agents or in cases when the use of drugs with a positive inotropic effect should be considered. The decision of the use of levosimendan was at the discretion of the treating physician.

The normality of the distribution was verified using Shapiro-Wilk test. Continuous variables are presented as median and interquartile range (IQR) or mean \pm standard deviation (SD), categorical variables as absolute frequencies and percentages. The comparison between two variables was performed with Mann Whitney U test or appropriate t-Student test according to the distribution. For comparison of more than two variables, Kruskal - Wallis test or ANOVA was used regarding the normality of data distribution. The two-sided $p$ value $<0.05$ was considered significant. STATISTICA version 13.1 was used to perform the analyses.

\section{Results}

The characteristics of the study groups are presented in Table 1. In the Levo group, there were significantly more patients with diabetes and arterial hypertension. This group also manifested significantly lower left ventricular ejection fraction together with a higher concentration of B-type natriuretic peptide.

The mean length of hospital stay for the ST patients was 9.4 days compared to 29.1 days for Levo group. The median total cost of hospitalization of patients receiving ST was significantly lower compared to Levo group [PLN 6,612.5 (IQR 3,624.9-13,301.3) vs PLN 23,854.9 (IQR 10,900.4-40,391.5), p < 0.001]. On the other hand, the median daily cost of hospitalization did not differ between the ST and Levo group [PLN 772 (616.4-1,629) vs PLN 1,010.5 (IQR 787.4-1,172), $\mathrm{p}=0.1]$. The mean cost of levosimendan acquisition was PLN 7,492.47. The mean cost of hospitalization before the drug administration was accounted for PLN $6,730.84$.

Treatment costs were compared depending on the length of hospitalization. Interestingly, the total cost of treatment was significantly lower only in the ST subgroup hospitalized for less than 2 weeks ( $p=0.008$ ). A detailed description of costs depending on the hospitalization period is presented in Table 2 .

When analyzed the Levo patients, the drug was administered on average on day 8.3 from the beginning of hospitalization. Thus, we compared the cost of hospitalization between the two subgroups: group $1(n=18)$ that received the drug up to the 8th day of hospitalization and group $2(n=11)$ received the drug 
Table 1. Study group characteristics

\begin{tabular}{lccc}
\hline & $\begin{array}{c}\text { Levosimendan, } \\
\mathbf{n = 2 9}\end{array}$ & $\begin{array}{c}\text { Standard therapy, } \\
\mathbf{n}=\mathbf{1 0 5 7}\end{array}$ & p-value \\
\hline Age, years (+/- SD) & $73.2(9.7)$ & $71(12.4)$ & 0.5 \\
Females, n (\%) & $7(24.1)$ & $230(21.7)$ & 0.7 \\
Arterial hypertension, n (\%) & $25(86.2)$ & $672(63.6)$ & 0.02 \\
Diabetes mellitus, n (\%) & $18(62.1)$ & $375(35.4)$ & 0.006 \\
History of infarction, PCl or CABG n (\%) & $21(72.4)$ & $720(68.7)$ & 0.6 \\
EF LV \%, median (IQR) & $25(20-33)$ & $31(27-38)$ & 0.003 \\
eGFR (estmated glomerular filtration rate) & $5(17.2)$ & $130(12.3)$ & 0.5 \\
< 30 mL/min/m², n (\%) & & & \\
BNP (B-type natriuretic peptide) pg/mL; median (IQR) & $1,513.4(874.0-2,415.0)$ & $758.7(252.2-1,944.75)$ & 0.01
\end{tabular}

Table 2. Total costs of hospitalization depending on the length of stay in the Levo group vs ST group. Total costs are presented as median (IQR)

\begin{tabular}{|c|c|c|c|c|c|}
\hline $\begin{array}{l}\text { Length of } \\
\text { hospitalization } \\
\text { (days) }\end{array}$ & $\begin{array}{l}\text { Levosimendan } \\
\text { (n) }\end{array}$ & $\begin{array}{l}\text { Levosimendan } \\
\text { - total cost, PLN }\end{array}$ & $\begin{array}{l}\text { Standard } \\
\text { therapy }(n)\end{array}$ & $\begin{array}{l}\text { Standard therapy } \\
\text { - total cost, PLN }\end{array}$ & p-value \\
\hline $0-14$ & 10 & $\begin{array}{c}9,933.2 \\
(7,916.8-12,743.9)\end{array}$ & 877 & $\begin{array}{c}5,439.9 \\
(2,861.2-10,141.8)\end{array}$ & 0.008 \\
\hline $15-30$ & 7 & $\begin{array}{c}16,289.1 \\
(14,693.9-22,522)\end{array}$ & 139 & $\begin{array}{c}13,582.3 \\
(10,489.0-22,407.8)\end{array}$ & 0.4 \\
\hline 31 and more & 12 & $\begin{array}{c}42,159.6 \\
(38,543.3-64,346.9)\end{array}$ & 41 & $\begin{array}{c}36,223.2 \\
(26,229.8-81,762.1)\end{array}$ & 0.3 \\
\hline
\end{tabular}

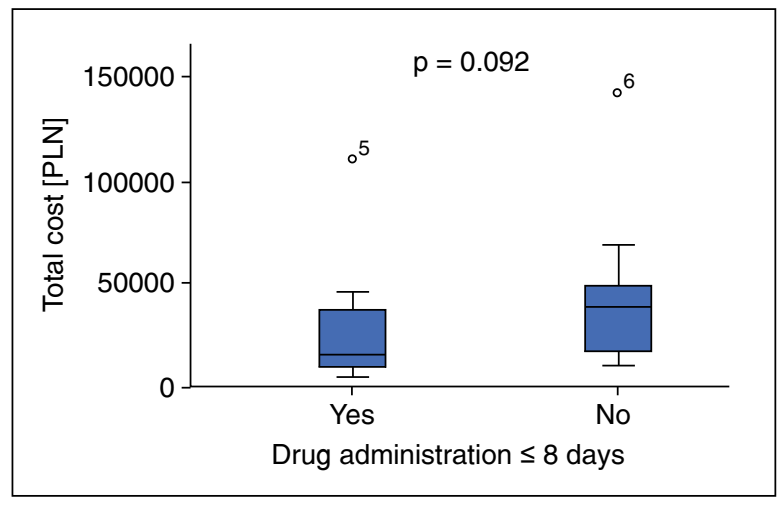

Figure 1. Total cost of hospitalization depending on the time of levosimendan administration (median with IQR)

after the 8th day of hospitalization. We have noticed a trend towards significantly lower total median cost of hospitalization in the group 1 vs the group 2 [PLN 16,057.9 (IQR 9,928.5-37,978.34) vs PLN 39,108.3 (IQR 17,375-49,556); $p=0,09$ ] (Fig. 1). The difference of median daily cost between these groups was not significant [PLN 1,033 (IQR 795-1,239) vs PLN 1,002 (IQR $721-1,081), p=0.4]$. The median length of hospital stay was significantly shorter in group 1 [14 (IQR 11-35) days vs 36 (IQR 22-53); $p=0.019$ ] (Fig. 2). Importantly, the

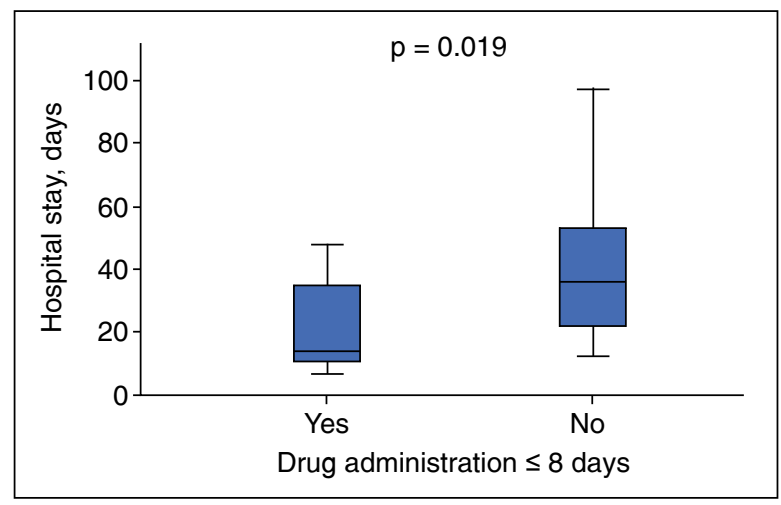

Figure 2. Length of hospital stay in reference to the time of levosimendan administration (median with IQR)

subgroups did not differ in baseline parameters, including age, ejection fraction and BNP concentration $(p=0.5)$.

\section{Discussion}

Our observation showed that in the Polish clinical setting and health care system the early administration of levosimendan in patients with HF exacerbation might be associated with favorable economic and health effects. 
In our analysis, the Levo group was hospitalized significantly longer when compared to the ST group, together with the higher total cost of therapy. In addition, we noticed a trend towards higher hospitalization costs in patients who received the drug later; these patients were also hospitalized longer. It must be noted that patients receiving levosimendan were more likely to be diabetic and hypertensive, they manifested more severe HF based on lower LV EF and higher BNP concentration. Such conditions and co-morbidities usually demand additional therapies and treatment modalities as well as are correlated with a longer hospital stay. Additionally, levosimendan in our group was added to treatment in situations where conventional therapy was insufficient, often as a last chance therapy. The abovementioned factors may contribute to an increase in resource utilisation and influence our results in reference to incremental costs of the therapy. It is supported by the fact that the daily cost of therapy did not differ between the groups.

Although the cost of levosimendan therapy was higher when the hospitalization lasted up to 2 weeks, in case of longer hospitalization the costs of ST and Levo were comparable. It suggests that if levosimendan was administered later and when the previous therapy was ineffective, this management relatively increased the total costs. The costs were derived not only from the drug itself but also from la onger hospital stay. The cost of treatment until levosimendan administration is also a significant part of the total cost of hospitalization. Therefore our observations support the positive effect of levosimendan both on the clinical and economic outcome and offer preliminary evidence of its cost-effectiveness. When the drug is administered earlier during the hospitalization, it influences the clinical improvement thus reducing the hospitalization period.

Our observations are consistent with other studies that addressed the economic issue and clinical benefit of levosimendan therapy. The LIDO trial showed that the use of levosimendan instead of dobutamine was associated with a reduction in mortality without an increase in the need of hospitalization [6]. Although the difference in absolute drug costs was relatively high, the cost per life-year saved was acceptable from a longer perspective [6]. The study of Fedele et al confirmed that the initial levosimendan treatment for HF was associated with a reduced duration of hospital stay and fewer re-hospitalizations [9]. In REVIVE II trial, patients treated with levosimendan had a shorter length of stay and lower cost for the initial hospital admission relative to patients treated with placebo [11]. Interestingly, Nieminen et al showed that the use of levosimendan for HF decompensation provided savings for hospitals driven by the reduction in the length of hospital stay in every European country considered in the study (Italy, Spain, Greece, Germany, Sweden, Finland, Israel) [12].
Our study is limited mainly by single-center setting, retrospective character and small sample size in levosimendan group. From the global economic perspective, another limitation is the lack of follow-up and rehospitalization rate, as we analyzed the only in-hospital cost of a single admission. Despite these limitations, our analysis contributes substantial insight into the pharmaco-economic issue of levosimendan therapy in Poland. Although levosimendan might increase costs of hospitalization it may also extend the benefits of treatment on the quality of life, rehospitalization rates and survival, thus reducing the global HF burden on the health care system.

In conclusion, early administered levosimendan in HF exacerbation seems to be cost-effective in Polish clinical and economic settings. We believe that the early introduction of levosimendan in HF therapy may reduce the total cost of hospitalization as well as shorten the hospital stay, despite the high cost of drug acquisition. Nevertheless, new trials or registry studies will be necessary to address this issue in Polish health care settings.

\section{Funding}

This research did not receive any specific grant from funding agencies in the public, commercial, or not-for-profit sectors.

\section{Disclosure of interest}

The authors report no conflicts of interest.

\section{References}

1. Bui AL, Horwich TB, Fonarow GC. Epidemiology and risk profile of heart failure. Nat Rev Cardiol. 2011; 8(1): 30-41, doi: 10.1038/nrcardio.2010.165, indexed in Pubmed: 21060326.

2. Heidenreich PA, Albert NM, Allen LA, et al. American Heart Association Advocacy Coordinating Committee, Council on Arteriosclerosis, Thrombosis and Vascular Biology, Council on Cardiovascular Radiology and Intervention, Council on Clinical Cardiology, Council on Epidemiology and Prevention, Stroke Council. Forecasting the impact of heart failure in the United States: a policy statement from the American Heart Association. Circ Heart Fail. 2013; 6(3): 606-619, doi: 10.1161/HHF.0b013e318291329a, indexed in Pubmed: 23616602.

3. Cook C, Cole G, Asaria P, et al. The annual global economic burden of heart failure. Int J Cardiol. 2014; 171(3): 368-376, doi: 10.1016/j. ijcard.2013.12.028, indexed in Pubmed: 24398230.

4. Papp Z, Édes I, Fruhwald S, et al. Levosimendan: molecular mechanisms and clinical implications: consensus of experts on the mechanisms of action of levosimendan. Int J Cardiol. 2012; 159(2): 82-87, doi: 10.1016/j.ijcard.2011.07.022, indexed in Pubmed: 21784540

5. Moiseyev VS, Põder P, Andrejevs N, et al. RUSSLAN Study Investigators. Safety and efficacy of a novel calcium sensitizer, levosimendan, in patients with left ventricular failure due to an acute myocardial infarction. A randomized, placebo-controlled, double-blind study (RUSSLAN). Eur Heart J. 2002; 23(18): 1422-1432, doi: 10.1053/euhj.2001.3158, indexed in Pubmed: 12208222

6. Follath $\mathrm{F}$, Cleland J, Just $\mathrm{H}$, et al. Efficacy and safety of intravenous levosimendan compared with dobutamine in severe low-output heart failure (the LIDO study): a randomised double-blind trial. The Lancet. 2002; 360(9328): 196-202, doi: 10.1016/s0140-6736(02)09455-2. 
7. Landoni G, Biondi-Zoccai G, Greco M, et al. Effects of levosimendan on mortality and hospitalization. A meta-analysis of randomized controlled studies. Crit Care Med. 2012; 40(2): 634-646, doi 10.1097/CCM .0b013e318232962a, indexed in Pubmed: 21963578.

8. Lucioni C, D'Ambrosi A, Mazzi S, et al. Economic evaluation of levosimendan versus dobutamine for the treatment of acute heart failure in Italy. Adv Ther. 2012; 29(12): 1037-1050, doi: 10.1007/s12325-012 0070-4, indexed in Pubmed: 23233357.

9. Fedele F, D'Ambrosi A, Bruno N, et al. Cost-effectiveness of levosimendan in patients with acute heart failure. J Cardiovasc Pharmacol. 2011; 58(4): 363-366, doi: 10.1097/FJC.0b013e318224e0a2, indexed in Pubmed: 21697728
10. de Lissovoy G, Fraeman K, Salon J, et al. The costs of treating acute heart failure: an economic analysis of the SURVIVE trial. J Med Econ. 2008; 11(3): 415-429, doi: 10.3111/13696990802291679, indexed in Pubmed: 19450096

11. de Lissovoy G, Fraeman K, Teerlink JR, et al. Hospital costs for treatment of acute heart failure: economic analysis of the REVIVE II study. Eur J Health Econ. 2010; 11(2): 185-193, doi: 10.1007/s10198-0090165-2, indexed in Pubmed: 19582491.

12. Nieminen MS, Buerke M, Parissis J, et al. Pharmaco-economics of levosimendan in cardiology: a European perspective. Int J Cardiol. 2015; 199: 337-341, doi: 10.1016/j.ijcard.2015.07.049, indexed in Pubmed: 26241640 